\title{
Mecanismo para Cooperação e Coligação de Veículos Baseado na Teoria dos Jogos para Transmissão de Vídeos em VANETs
}

\author{
Wellington Lobato Junior ${ }^{1}$, Denis Rosário ${ }^{1}$, Leandro A. Villas ${ }^{2}$ e Eduardo Cerqueira ${ }^{1}$ \\ ${ }^{1}$ Universidade Federal do Pará (UFPA) \\ ${ }^{2}$ Universidade Estadual de Campinas (UNICAMP) \\ \{wellingtonl, denis\}@ufpa.br, leandro@ic.unicamp.br, cerqueira@ufpa.br
}

\begin{abstract}
Vehicular Ad-hoc NETworks (VANETs) promise a wide scope of multimedia services ranging from security and traffic announcements to entertainment and advertising videos. However, the transmission of real time videos over VANET must consider the Quality of Experience ( $Q o E)$ requirements, and also the dynamic network topology with short contact time. To mitigate such problems, neighbors vehicles moving in the same direction and wishing to cooperate may collaborate to form a platoon, and thus such vehicles move with similar speed. This paper introduces a platoon mechanism based on game theory for video transmission with QoE support, called of TJ2P. TJ2P mechanism selects vehicles to form a platoon with similar speeds and appropriate distance to transmit videos. Simulation results confirm the efficiency of the TJ2P mechanism to ensure video transmission with adequate QoE, without increasing the fuel consumption and $\mathrm{CO}_{2}$ emission compared to BLR and XLinGO protocols.
\end{abstract}

Resumo. As redes veiculares (VANETs) oferecem uma ampla gama de serviços multimídia que vão de segurança e avisos de tráfego a vídeos de entretenimento e publicidade. Contudo, a transmissão de vídeo em tempo real em uma VANET tem rigorosos requisitos de Qualidade de Experiência (QoE), além de topologia de rede altamente dinâmica com baixo tempo de contato. Para mitigar tais problemas, os veículos próximos que se deslocam no mesmo sentido e que desejam cooperar podem agrupar-se para formar um pelotão e, assim, os veículos irão movimentar-se com velocidade semelhante. Nesse contexto, este artigo apresenta um mecanismo baseado em teoria dos jogos para formação de pelotão denominado de TJ2P, o qual possibilita a transmissão de vídeo com suporte a QoE. O mecanismo TJ2P seleciona veículos com velocidades similares e uma distância apropriada para formar o pelotão. Resultados de simulações constatam a eficiência do mecanismo TJ2P em garantir a transmissão de vídeo com QoE adequado, sem aumentar o consumo de combustível e emissão de $\mathrm{CO}_{2}$, em comparação aos protocolos BLR e XLinGO.

\section{Introdução}

De acordo com [Cisco 2016], a transmissão de dados multimídia irá representar até 90\% do tráfego dos dados globais. Em particular, [Ericsson 2013] mostra que a transmissão de multimídia por intermédio da rede sem fio tem tido o maior e mais rápido crescimento do segmento e representará cerca de 50\% do tráfego global de dados até 2019. Logo, os usuários irão produzir, compartilhar e consumir serviços de vídeo em tempo real até 
mesmo em seus veículos. Nesse contexto, as VANETs são redes que consideram veículos em movimento para criar uma rede ad-hoc e possibilitar uma diversidade de aplicações que vão de segurança e avisos de tráfego a vídeos de entretenimento e publicidade [Lee et al. 2014]. Por exemplo, os veículos podem disseminar vídeos de situações perigosas em uma estrada para informar visualmente motoristas e equipes de resgate sobre $o$ ocorrido.

Em uma transmissão de vídeo em VANET, os usuários esperam receber o conteúdo de vídeo com um nível adequado de Qualidade de Experiência (QoE), não importando as condições da VANET [Liotou et al. 2015]. Por exemplo, os usuários esperam receber vídeos em tempo real com boa qualidade, mesmo que estejam localizados a quilômetros de distância do acidente. Dessa forma, diversos protocolos de roteamento para a comunicação de Veículo para Veículo (V2V) foram propostos para atender à exigência de entrega vídeo em VANET [Liu et al. 2016]. Contudo, a transmissão de vídeo com suporte a QoE em uma VANET é uma tarefa difícil devido às frequentes desconexões causadas pelas mobilidades dos veículos [Quadros et al. 2015]. Por exemplo, [Uppoor and Fiore 2012] analisou um registro de mobilidade em uma rodovia da cidade de Colônia na Alemanha e concluiu que a maioria dos veículos têm contatos extremamente curtos, variando entre 1 e 15 segundos.

Para aumentar o tempo de contato entre veículos, estes podem ser agrupados com interesse em comum para que um veículo siga o outro, formando um pelotão, ou seja, mantendo a mesma distância e velocidade, evitando, assim, falhas de comunicação [Jia et al. 2014]. A movimentação de veículos em pelotão em uma rodovia traz benefícios para a disseminação e compartilhamento de vídeo, pois tais veículos podem ser usados para encaminhar os pacotes de vídeo [Amoozadeh et al. 2015]. E também, formação de pelotão melhora a vazão de veículos na rodovia, reduz o consumo de combustível e emissão de $\mathrm{CO}_{2}$ [Jia et al. 2014]. Entretanto, os veículos terão o tempo de viagem alterado devido às mudanças de velocidade para participarem do pelotão. Sendo então necessário oferecer benefícios aos veículos participantes do pelotão.

A formação de pelotão conta com a participação ativa dos veículos/usuários, onde mecanismos de incentivo fomentam uma maior participação dos mesmos [Wang and Lin 2015]. Por exemplo, um veículo que participa do pelotão poderá ter prioridade na transmissão de conteúdo no futuro ou participar de pelotão composto por veículos com melhores reputações. Os regimes monetários, recompensas, cupons, moedas virtuais ou créditos são os métodos diretos para bonificar os veículos que participam do pelotão. No entanto, faz-se necessário escolher os veículos que possibilitem disseminação de vídeos com QoE adequado, além de resolver situações de conflitos de um veículo desejando participar de dois ou mais pelotões. Diante desse quadro, a teoria dos jogos provê um conjunto de ferramentas matemáticas e técnicas para a modelagem de situações que envolvem conflito de interesses entre os agentes de decisão.

As principais abordagens envolvendo teoria dos jogos são subdivididas em jogos cooperativos e jogos não cooperativos [Hu et al. 2016]. Nos jogos não cooperativos, os jogadores não podem estabelecer compromissos antes de iniciar o jogo. Por outro lado, nos jogos cooperativos, os jogadores podem estabelecer relações prévias antes de começar o jogo, além de estabelecer compromissos garantidos com os demais jogadores. Nos jogos simultâneos, o processo de interação do jogo acontece em uma única etapa, já nos jogos 
sequenciais a interação acontece em uma sequência de etapas. Portanto, na formação de um pelotão, considerando teoria dos jogos, é esperado que cada jogador (veículo) escolha sua estratégia sem ter acesso à estratégia e sem esperar a decisão dos outros jogadores, ou seja, um jogo não cooperativo e simultâneo.

Este artigo propõe um mecanismo para formação de Pelotão baseado na teoria dos jogos para que seja realizada transmissão de vídeo com suporte a QoE, denominado de TJ2P(Teoria dos Jogos to Platoon). O mecanismo TJ2P considera teoria dos jogos para decidir quais veículos devem participar do pelotão e resolver situações de conflitos entre dois ou mais coligações durante a transmissão. A cooperação entre os veículos é garantida por meio de uma função de recompensa, a qual garante uma retribuição para os participantes do pelotão. O TJ2P seleciona, para participar do pelotão, um conjunto de veículos que estejam em movimento no mesmo sentido, com velocidades semelhantes e uma distância apropriada para disseminar o vídeo, provendo dessa maneira uma maior conectividade entre os veículos. Resultados de simulações constatam a eficiência do mecanismo TJ2P em transmitir vídeo com QoE 39\% maior que os vídeos transmitidos pelos protocolos Beacon-Less Routing (BLR) e Cross-layer Link Quality and Geographic-aware beaconless Opportunistic Routing (XLinGO). O mecanismo TJ2P forneceu uma recompensa aos veículos por terem aumentado o seu tempo de viagem,e também diminuiu o consumo de combustível e emissão de $\mathrm{CO}_{2}$ comparado aos protocolos BLR e XLinGO.

As seções deste artigo estão organizadas da seguinte forma: A Seção 2 discute os principais trabalhos envolvendo disseminação de vídeo em VANET. A Seção 3 descreve o modelo de rede e o mecanismo TJ2P. A Seção 4 descreve os resultados obtidos com as simulações. A Seção 5 conclui o trabalho e apresenta os trabalhos futuros.

\section{Trabalhos Relacionados}

Diversos protocolos de roteamento foram propostos para atender à exigência de entregar vídeo em VANETs. [Braun et al. 2010] introduzem o conceito de Dynamic Forwarding Delay (DFD) como a decisão de encaminhamento no protocolo BLR. Ao invés de realizar uma troca contínua de informações com os nós vizinhos, cada nó faz a decisão de encaminhamento calculando o valor DFD com base na sua localização atual e na localização do vizinho contida no pacote recebido. Todos os receptores definem um temporizador com base no valor de DFD e aquele que terminar o temporizador primeiro será selecionado como o nó encaminhador. [Rosário et al. 2014] propõem o protocolo XLinGO, o qual considera parâmetros de múltiplas camadas para cálculo de DFD, tais como tamanho do buffer, qualidade do enlace, localização e energia. [Quadros et al. 2015] apresentam um mecanismo para calcular o DFD baseado em parâmetros do vídeo, localização e QoE. Contudo, uma VANET tem topologia de rede altamente dinâmica com baixo tempo de contato, ocasionando frequentes desconexões. Além disso, tais protocolos de roteamento não levam em consideração formação de pelotão para reduzir atrasos e perdas de pacotes durante a transmissão.

Em relação a formação de pelotão em VANET, [Zhang and Cao 2011] propuseram dois algoritmos baseados em custo e eficiência com o intuito de encontrar o melhor veículo para transmitir dados dentro do pelotão e analisar possíveis anomalias na mobilidade que possam afetar a transmissão dos dados. [Jia et al. 2014] apresentam um modelo de mobilidade baseado em pelotão levando em consideração os parâmetros de mobilidade 
dos carros, tais como fluxo de tráfego, velocidade de coligação, tamanho da coligação e faixa de transmissão. Porém, este trabalho considera que todos os veículos do pelotão podem comunicar-se diretamente, isso significa que o comprimento do pelotão não excede um salto. Além do mais, os mecanismos para formação de pelotão devem fornecer incentivos para os veículos que participam do pelotão, uma vez que os veículos terão o tempo de viagem, consumo de combustível e emissão de $\mathrm{CO}_{2}$ alterados.

[Chen et al. 2011] propõem um mecanismo de incentivo baseado em teoria dos jogos para formar grupos. Tal mecanismo pode ser utilizado na tomada de decisão de protocolos de roteamento, estimulando a cooperação entre os participantes da rede. $\mathrm{O}$ mecanismo considera o espaço de armazenamento de cada veículo da rede para decidir quais pacotes podem ser descartados e quais veículos podem participar de uma transmissão. [Gerla et al. 2014] expõem uma abordagem utilizando teoria dos jogos para incentivar a participação dos nós intermediários a realizarem networking coding. Contudo, tais trabalhos não consideram a maneira como ocorre a coligação dos nós para formar um pelotão.

Com base na análise dos trabalhos relacionados, pode-se concluir que é essencial considerar um mecanismo de coligação eficiente para formação de um pelotão, como forma de reduzir o número de desconexões causadas pela alta mobilidade dos veículos. Isso requer ciência acerca dos requisitos QoE e considerar mecanismos de incentivo para recompensar os veículos que participam do pelotão.

\section{Mecanismo Baseado em Teoria dos Jogos para Formação de Pelotão Visando Transmissão de Vídeo em uma VANET (TJ2P)}

Esta seção descreve o mecanismo TJ2P, o qual considera teoria dos jogos para a formação do pelotão entre os veículos de uma VANET. Para formar o pelotão, o mecanismo proposto seleciona veículos que estejam movimentando-se na mesma direção, tenham velocidades semelhantes e uma distância apropriada para transmissão do vídeo com qualidade. Tais veículos recebem uma recompensa por participarem do pelotão. Á vista disso, primeiramente será apresentado o modelo de sistema e rede, em seguida, será feita a descrição do mecanismo TJ2P.

\subsection{Modelagem do Sistema e Rede}

Foi considerado um ambiente de VANET para disseminação de vídeos de acidentes, onde existem $n$ veículos (nós de uma VANET) em movimento em uma rodovia com $j$ pistas e cada veículo tem uma identidade individual denotada por $(i \in[1, n])$. Estes nós são representados por um grafo dinâmico $G(V, E)$, onde os vértices $V=\left\{v_{1}, \cdots, v_{n}\right\}$ representam um conjunto finito de veículos e as arestas $E=\left\{e_{1}, \cdots, e_{m}\right\}$ formam um conjunto de enlaces de comunicação sem fio assimétrico entre os veículos vizinhos $N\left(v_{i}\right) \subset V$. Os vizinhos a 1 salto de distância de $v_{i}$ são representadoss por $N\left(v_{i}\right)$.

Cada veículo $\left(v_{i}\right)$ movimenta-se em uma das pistas da rodovia com uma direção $\left(\overrightarrow{d r_{i}}\right)$ e uma velocidade $S_{i}$ variando entre os limites mínimo $\left(S_{\min }\right)$ e máximo $\left(S_{\max }\right)$. Cada veículo $\left(v_{i}\right)$ é ciente de sua própria localização $L_{i}(x, y)$ por meio de sistema de posicionamento, tal como o GPS. Por outro lado, a localização do nó destino $(D N) \subset V$ é conhecida por algum serviço de localização. Cada veículo $v_{i}$ é equipado com um transmissor de rádio compatível com o padrão IEEE 802.11 p, por meio do qual os veículos $v_{i}$ 
comunicam-se com seus vizinhos $N\left(v_{i}\right)$. Tais nós têm uma fila $(Q)$ com uma capacidade máxima $\left(Q_{\max }\right)$ e um tamanho atual da fila $\left(Q_{\text {length }}\right)$. A política de escalonamento de transmissão de pacotes é realizada pelo algoritmo First in First Out (FIFO) e o descarte de pacotes utiliza o algoritmo Drop Tail. Os veículos também têm um codificador e decodificador de vídeo. Por conveniência de notações, foi denotado $S N \subset V$ como o veículo $v_{i}$ responsável por capturar o fluxo de vídeo e transmitir para o destino $D N$ por meio de múltiplos veículos intermediários $\left(F_{i} \subset V\right)$.

O mecanismo TJ2P tem duas etapas para a formação do pelotão. A primeira etapa (comunicação) consiste em disseminar informações para selecionar veículos que estejam em movimento na mesma direção, tenham velocidade semelhante e uma distância apropriada para transmissão do vídeo com qualidade. A segunda etapa (mobilidade) é responsável por criar e manter um pelotão para disseminação do vídeo. Ainda sobre o mecanismo TJ2P, observam-se as seguintes premissas.

- O jogo $J$ é não cooperativo e simultâneo.

- Não existem nós mal intencionados na rede.

- Todos os veículos $v_{i}$ estão dispostos a participar do pelotão.

\subsection{Configurações do Jogo para o Mecanismo TJ2P}

Os veículos $v_{i}$ são jogadores de um jogo $J$ não cooperativo e simultâneo, pois os veículos $v_{i}$ escolhem suas estratégias sem esperar e sem ter acesso às estratégias dos seus vizinhos $N\left(v_{i}\right)$. Formalmente, pode-se descrever o jogo $J$ como $\{V, A, U\}$. O conjunto de veículos é representado por $V$, o conjunto de estratégias disponíveis para cada veículo $v_{i}$ é denotado por $A=\left\{a_{1}, a_{2}, a_{3}, \ldots a_{n}\right\}$, ou seja, $a_{i} \in\{$ Agrupar, Não-agrupar $\}$. O veículo $v_{i}$ que decidir pela estratégia $a_{i}$ de Agrupar para participar do pelotão terá o tempo de viagem, consumo de combustível e emissão de $\mathrm{CO}_{2}$ alterados. Tal veículo receberá uma recompensa $u_{i}$ por participar do pelotão. $U=\left\{u_{1}, u_{2}, u_{3}, \ldots u_{n}\right\}$ representa o conjunto com as funções de recompensa de cada veículo $v_{i}$, dependendo do conjunto de estratégias $A$.

Um dado veículo $v_{i}$ receberá uma recompensa $u_{i}$ e terá um custo $C$ para a estratégia $a_{i}$ de agrupar. $\mathrm{O}$ custo $C$ está relacionado à utilização de recursos da rede, consumo de gasolina, emissão de $\mathrm{CO}_{2}$ e alteração no tempo de viagem. Por outro lado, um dado veículo $v_{i}$ terá zero de recompensa $u_{i}$, caso decida pela estratégia $a_{i}$ de não-agrupar, pois não será possível formar o pelotão para transmitir o vídeo. Nesse sentido, faz-se necessário que os veículos estejam dispostos a agrupar-se para formar um pelotão, isto é, os veículos $v_{i}$ devem escolher pela estratégia $a_{i}$ de agrupar.

O Algoritmo 1 apresenta operações do mecanismo TJ2P para que os veículos de uma VANET formem um pelotão. O mecanismo TJ2P é considerado reativo, pois os veículos do pelotão são selecionados no mesmo instante em que um veículo $v_{i}$ captura e dissemina um vídeo de acidente. Esse veículo $v_{i}$ será considerado como fonte $S N$, pois terá que enviar um vídeo para um veículo de destino $D N$ por meio de múltiplos veículos intermediários $F_{i}$. Para a formação do pelotão, os veículos transmitem três tipos de mensagens de controle: advertisement (adv), join e acknowledgment (ack).

Na etapa de comunicação, o veículo $S N$ envia uma mensagem de $a d v$ aos seus vizinhos $N(S N)$, para iniciar a formação do pelotão. A mensagem de adv contém informações de velocidade $S_{S N}$ e localização $L_{S N}(x, y)$ do veículo $S N$ (linhas 1-4 do 
Algoritmo 1). Essas informações são utilizadas para calcular a recompensa $u_{i}$ para um dado veículo $v_{i}$ participar do pelotão. Após o envio da mensagem de $a d v$, o veículo $S N$ aguarda pelas mensagens de join enviadas por seus vizinhos $N(S N)$.

Ao receber uma mensagem de $a d v$, os veículos $N(S N)$ devem verificar se estão dentro da zona de encaminhamento do veículo $S N$. Um veículo $v_{i}$ está dentro da zona de encaminhamento, caso esteja localizado entre os veículos $S N$ e $D N$, bem como se movendo na mesma direção $\overrightarrow{d i r_{S N}}$ do veículo $S N$. Os veículos $v_{i}$, dentro da zona de encaminhamento, decidem por uma das estratégias $a_{i}$ com base na recompensa para cada estratégia. O valor da recompensa $u_{i}$ é calculado considerando as informações de velocidade do veículo fonte $S_{S N}$, a sua própria velocidade $S_{i}$, a distância euclidiana ( $\operatorname{dist}_{S N, v_{i}}$ ) entre $S N$ e $v_{i}$ e um ganho aleatório $\Omega_{i} \in\left[\Omega_{\min }, \Omega_{\max }\right]$ por participar do pelotão, esse ganho proposto pode representar uma bonificação monetária por garantir melhorias na rede. A função de recompensa $u_{i}$ é calculada de acordo com a seguinte fórmula:

$$
u_{i}=\left(S_{i}-S_{S N}\right)+\operatorname{dist}_{S N, v_{i}}+\Omega_{i}
$$

O veículo $v_{i}$ que decidir pela estratégia $a_{i}$ de agrupar deve enviar uma mensagem de join para o veículo $S N$, a fim de informar o valor da sua recompensa $u_{i}$ para participar do pelotão. Tal veículo $v_{i}$ deve armazenar o valor da velocidade $S_{S N}$, pois, $v_{i}$ deve movimentar-se com a mesma velocidade do veículo $S N$, caso participe do pelotão (linhas 7-12 do Algoritmo 1).

A cada recepção de join, o veículo $S N$ armazena o valor da função de utilidade (UF) (Eq. 2) e da identificação do veículo $v_{i}$ que deseja participar do pelotão em uma lista de candidatos $C$ (linhas 13-15 do Algoritmo 1). Tão logo o procedimento de envio de join seja finalizado, o veículo $S N$ escolhe o veículo $v_{i}$ com menor valor de $U F$ para realizar a coligação, pois o mecanismo TJ2P considera que tal veículo $v_{i}$ tem a recompensa $u_{i}$ mais próxima da recompensa mínima $u_{\text {min }}$. Para o cálculo da recompensa mínima $u_{m i n}$, foi considerado ganho $\Omega_{i}$ zero as velocidades $S_{i}$ e $S_{S N}$ iguais e distância dist ${ }_{S N, v_{i}}$ de $2 / 3$ do raio de alcance $R_{\max }$. Com base em resultados de simulação, essa distância é considerada apropriada para transmissão do vídeo com qualidade, pois distâncias menores aumentam a quantidade de saltos. Enquanto que, distâncias maiores causam desconexões, visto que o raio de alcance $R_{\max }$ varia devido a problemas de atenuação e sombreamento do canal sem fio. Portanto, o mecanismo TJ2P seleciona veículos $v_{i}$ para participar do pelotão com velocidades semelhantes com a do pelotão e uma distância apropriada para transmissão do vídeo. Dessa forma, o mecanismo TJ2P provê disseminação de vídeo com QoE adequado, ainda evita que os veículos participantes do pelotão não tenham o tempo de viagem, o consumo de combustível e a emissão de $\mathrm{CO}_{2}$ grandemente alterados.

$$
U F=\min _{i \in C}\left\{\left|u_{i}-u_{\min }\right|\right\}
$$

O veículo $S N$ envia uma mensagem de ack para o veículo $v_{i}$ escolhido para participar do pelotão (linhas 5-6 do Algoritmo 1). Os veículos do pelotão atuam como encaminhadores $F_{i}$ dos pacotes de vídeo enviados do veículo $S N$ para o destino $D N$, e, assim, colaboram em uma transmissão do vídeo com menor número de desconexões. Uma vez recebida a mensagem de $a c k$, o veículo $v_{i}$ muda para a etapa de mobilidade 
alterando a sua velocidade $S_{i}$ para a velocidade do pelotão $S_{S N}$, como forma de manter uma distância fixa com $S N$ e formar um pelotão. O veículo $v_{i}$ passa a ser o novo líder do pelotão, i.e., enquanto que o veículo $S N$ continua com o processo de coligação até que o veículo de destino $D N$ seja alcançado (linhas 16-18 do Algoritmo 1). Vale a pena ressaltar que existem retransmissões das mensagens de $a d v$, join e $a c k$, caso elas sejam perdidas.

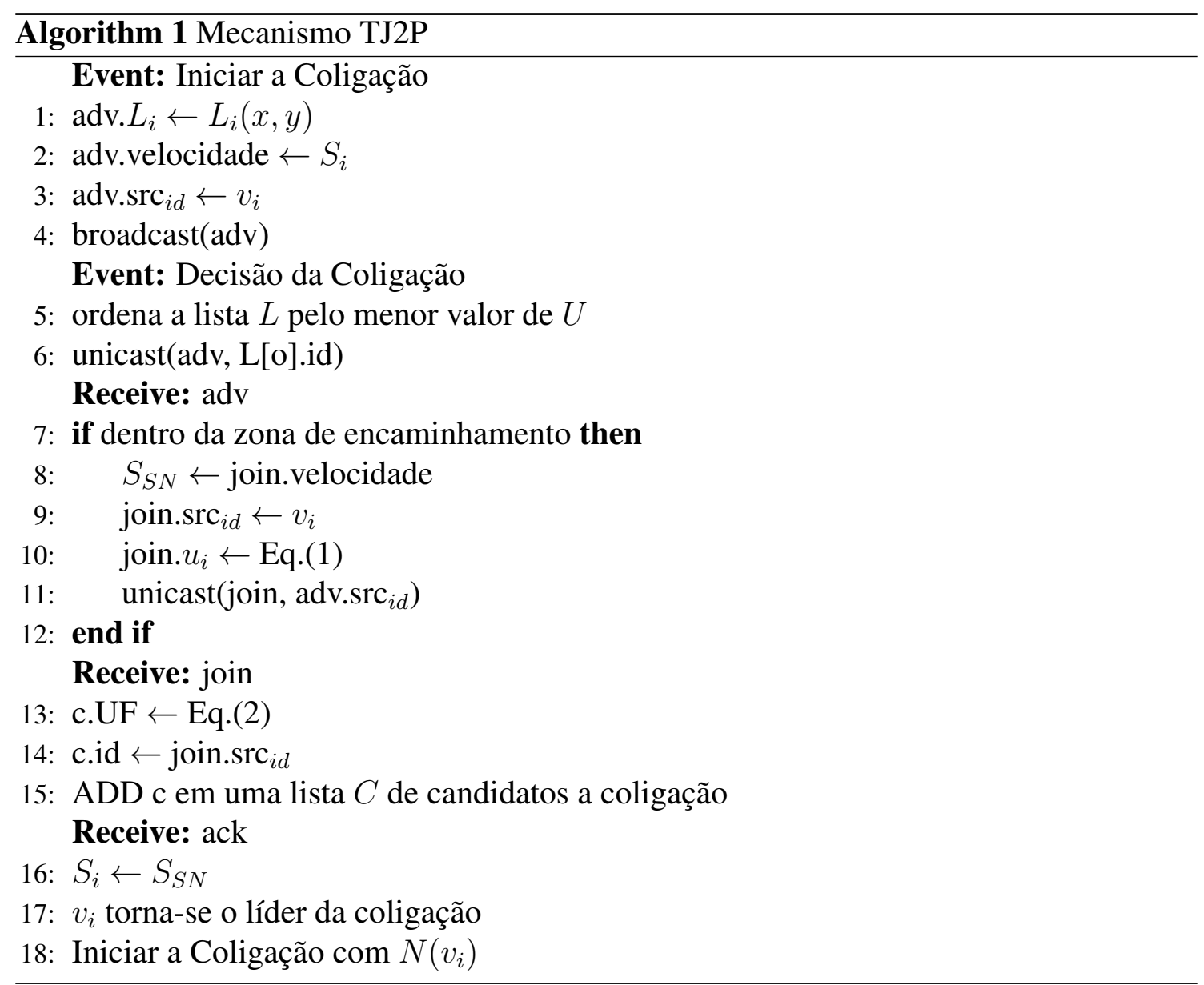

\subsection{Estudo de Caso}

A Figura 1 apresenta um estudo de caso para formação de pelotão considerando o mecanismo TJ2P. Esse estudo de caso considera parte de uma VANET formada pelos nós $v_{1}$, $v_{2}, v_{3}, v_{4}, v_{5}, v_{6}$ e $v_{7}$ movimentando-se em uma rodovia. Nesse estudo de caso, $v_{1}$ é considerado como o veículo fonte $S N$ e $v_{7}$ é considerado como o veículo de destino $D N$. Os nós têm um raio de transmissão $R_{\max }$ de 250 metros (m) e uma distância dist ${ }_{S N, v_{i}}$ considerada adequada é de $166.6 \mathrm{~m}$. Os veículos estão localizados em $L_{1}(50,10), L_{2}(100,25)$, $L_{3}(150,10), L_{4}(250,5), L_{5}(25,25), L_{6}(125,0), L_{7}(400,10)$ e movimentando-se a uma velocidade de $20,10,30,20,25,30$ e $25 \mathrm{~m} / \mathrm{s}$, respectivamente. A lista de candidatos de $v_{1}$ armazena as informações de $v_{i}, u_{i}$ e UF.

Seguindo o funcionamento do TJ2P, o veículo $v_{1}$ inicia o processo de coligação enviando a mensagem de $a d v$ contendo a sua localização $L_{i}$ e velocidade $S_{i}$, conforme ilustrado na Figura 1(a). Os veículos $v_{2}, v_{3}, v_{4}, v_{5}$ e $v_{6}$ recebem a mensagem de $a d v$. 
Porém, os veículos $v_{5}$ e $v_{6}$ estão fora da zona de encaminhamento de $v_{1}$, pois $v_{5}$ está mais distante de $v_{7}$, comparado a $v_{1}$ e $v_{6}$ está movimentando-se em direção $\overrightarrow{d i r_{i}}$, oposta a $v_{1}$. Logo, $v_{5}$ e $v_{6}$ não podem participar do pelotão.

Os veículos $v_{2}, v_{3}$ e $v_{4}$, que estão dentro da zona de encaminhamento, escolhem uma recompensa aleatória $\Omega_{i}$, i.e., 20, 25 e 10, respectivamente e após isso calculam o valor da recompensa $u_{i}$. Tais veículos enviam uma mensagem de join para $v_{1}$ contendo o valor da recompensa $u_{i}$, ou seja, $92.2,125$ e 220.06, respectivamente, conforme ilustra a Figura 1(b). O veículo $v_{1}$ recebe as mensagens de join e calcula o valor de UF para os veículos $v_{2}, v_{3}$ e $v_{4}$, ou seja, 74.4, 41.6 e 53.46, respectivamente. Na Figura 1(c), o veículo $v_{1}$ envia a mensagem de $a c k$ para o veículo $v_{3}$, uma vez que $v_{3}$ tem a recompensa $u_{3}$ mais próximo da recompensa mínima $u_{\text {min }}$, ou seja, $v_{3}$ tem a velocidade mais próxima da velocidade de $v_{1}$ e uma distância mais próxima da distância adequada para transmissão de vídeo com qualidade. Ao receber a mensagem de $a c k, v_{3}$ passa a movimentar-se a 20 $\mathrm{m} / \mathrm{s}$, formando, assim, o pelotão com $v_{1}$. O processo de coligação segue até alcançar o veículo destino $v_{7}$.
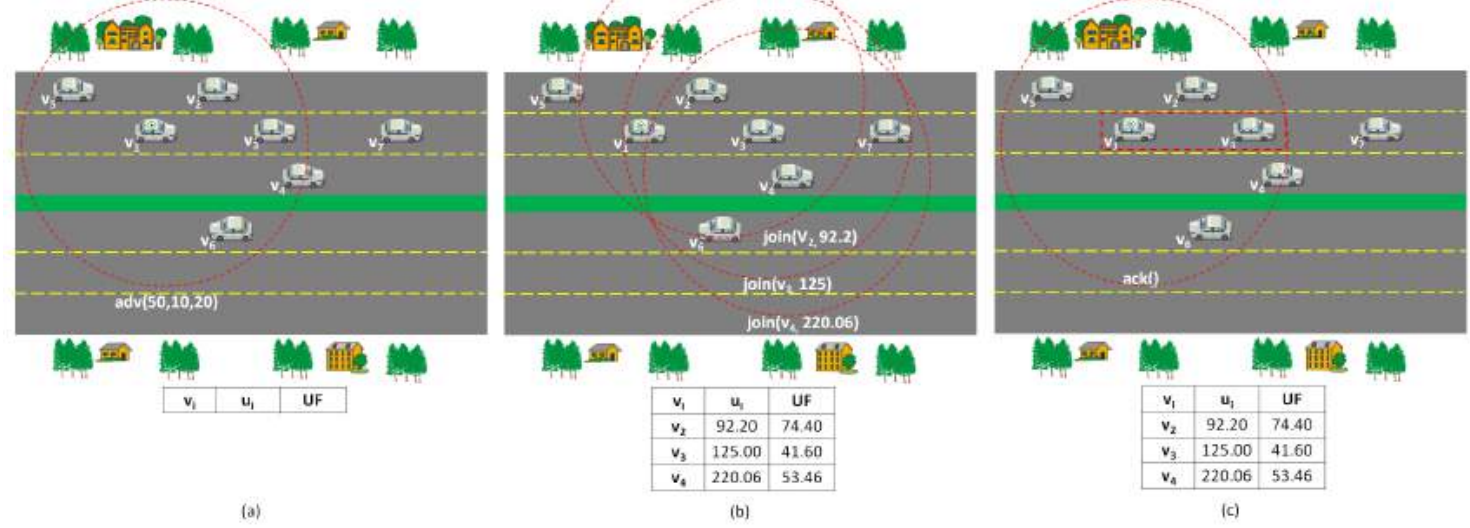

Figura 1. Estudo de Caso: Formação do Pelotão Entre os Veículos de uma VANET

\section{Avaliação}

Esta seção descreve a metodologia e métricas utilizadas para avaliar a qualidade dos vídeos transmitidos em uma VANET, bem como o impacto no consumo de combustível, emissão de $\mathrm{CO}_{2}$ e atraso na viagem. Foram transmitidos vídeos com diferentes complexidades, e com o veículo fonte $S N$ localizado em diferentes distâncias para o veículo $D N$. Dessa forma foi possível analisar o impacto na qualidade dos vídeos transmitidos pelo mecanismo TJ2P comparado aos protocolos BLR e XLinGO.

\subsection{Descrição do Cenário e das Métricas}

As simulações foram realizadas no framework M3WSN do OMNeT $++{ }^{1}$. Para estabelecer um cenário relevante, utilizou-se $12 \mathrm{Km}$ da rodovia de San Diego do OpenStreetMap ${ }^{2}$, a qual foi importada pelo SUMO (Simulation of Urban MObility) para gerar os registros

\footnotetext{
${ }^{1}$ http://www.omnetpp.org

${ }^{2} \mathrm{http}: / /$ www.openstreetmap.org/
} 
de movimentação dos veículos. Isso permite reproduzir os movimentos dos veículos e as interações V2V, de acordo com dados empíricos. As simulações foram repetidas 33 vezes com diferentes sementes de aleatoriedade e os resultados apresentam os valores com um intervalo de confiança de 95\%. As simulações foram executadas durante 800 segundos, sendo que os vídeos foram transmitidos a partir dos 100 primeiros segundos até o tempo de simulação de 700 segundos. Decidiu-se organizar a arquitetura da simulação para permitir que existam variações temporais, enlaces assimétricos e raio de alcance irregular, tal como é esperado em um cenário de VANET.

Os veículos movem-se com uma velocidade variando de 20 a $45 \mathrm{~m} / \mathrm{s}$, onde cada veículos possui um rádio IEEE $802.11 \mathrm{p}(5.89 \mathrm{GHz}, 6 \mathrm{Mbps})$ com um raio de transmissão $R_{\max }$ de $250 \mathrm{~m}$. Seguindo a abordagem proposta em [Torres et al. 2015], situações de acidente foram agendadas para que o veículo $D N$ realize requisições de vídeo para o $S N$ próximo ao acidente. Os vídeos devem ser recebidos pelo veículo de destino $D N$ a uma distância inferior a $2.0 \mathrm{~km}$ do veículo $S N$, fornecendo um limite de salto conforme apresentado em [Di Felice et al. 2013].

Os vídeos transmitidos nas simulações têm diferentes características de mobilidade e complexidade, os quais foram baixados da biblioteca [Library 2017]. Com duração de 10 segundos codificados em H.264 com 300 kbps, 30 quadros por segundo e com 352 x 288 pixels. O decodificador de vídeo no veículo de destino $D N$ considera o método Frame-Copy para ocultar erro ao substituir cada quadro de vídeo perdido com o último recebido, reduzindo, assim, o impacto da perda de quadros e mantendo a qualidade de vídeo. A Tabela 1 sumariza os parâmetros de simulação. Foram realizadas simulações para demonstrar o impacto do mecanismo TJ2P comparando o desempenho com os protocolos BLR [Braun et al. 2010] e XLinGO [Rosário et al. 2014] em termos de qualidade dos vídeos transmitidos, consumo de combustível, emissão de $\mathrm{CO}_{2}$ e atraso na viagem.

Tabela 1. Parâmetros de Simulação

\begin{tabular}{r|c}
\hline Parâmetros & Valores \\
\hline \hline Área de Simulação & Rodovia com 12Km e 5 vias \\
Tempo de Simulação & 800 segundos \\
Velocidade dos Veículos & 20 à $45 \mathrm{~m} / \mathrm{s}$ \\
Quantidade de veículos & 400 \\
Rádio de Transmissão & IEEE $802.11 \mathrm{p}$ \\
Raio de Transmissão $R_{\max }$ & $250 \mathrm{~m}$ \\
Vídeos & Akiyo, Coastguard, Container, \\
& Hall e Mobile \\
Codificação dos Vídeos & H.264 \\
\hline
\end{tabular}

Em termos de avaliação de qualidade de vídeo, as métricas de Qualidade de Serviço (QoS) por si só não são suficientes para avaliar o nível de qualidade das aplicações multimídia. Isso se deve ao fato de que elas falham na captura de aspectos subjetivos de conteúdo de vídeo relacionados com a experiência humana [Liotou et al. 2015]. As métricas de QoE superam essas limitações e, dessa forma, foram consideradas as métricas de QoE objetiva bem conhecidas para avaliar a qualidade dos vídeos transmitidos, nomeadamente SSIM (Structural SIMilarity) e VQM (Video Quality Metric). SSIM $\in[0,1]$ 
avalia quadro a quadro do vídeo três componentes: luminância, contraste e similaridade estrutural. Quanto maior for o valor SSIM melhor será a qualidade de vídeo. O VQM $\in$ $[0,4]$ avalia danos na percepção do vídeo recebido com base em características do sistema visual humano, incluindo blurring, ruído e distorção de cores. Por outro lado, valores de VQM próximos de 0 significa vídeo com melhor qualidade. Foi utilizado o MSU Video Quality Measurement Tool (VQMT) para medir o valor SSIM e VQM de cada vídeo transmitido.

Para o cálculo de emissão de $\mathrm{CO}_{2}$ e consumo de combustível, foi utilizado o modelo EMIT integrado M3WSN. O EMIT é um modelo estatístico para calcular emissões instantâneas de $\mathrm{CO}_{2}$ e consumo de combustível baseado na aceleração e velocidade dos veículos, o qual é derivado da fórmula HBEFA ${ }^{3}$ - Handbook Emission Factors for Road Transport. Dessa forma, foi calculada a média do consumo de combustível e emissão de $\mathrm{CO}_{2}$ para os veículos que participaram da transmissão de vídeo.

\subsection{Analise dos Resultados}

A Figura 2 apresenta a média do SSIM para os vídeos transmitidos em diferentes distâncias entre os veículos $S N$ e $D N$ pelo TJ2P, BLR e XLinGO. Pode-se observar que o mecanismo TJ2P provê disseminação de vídeo com SSIM 39\% e 50\% melhor em comparação ao SSIM dos vídeos transmitidos pelo XLinGO e BLR, respectivamente. Esse ganho de SSIM para o mecanismo TJ2P pode ser explicado pela baixa taxa de perda de quadros em comparação com as transmissões feitas pelos protocolos XLinGO e BLR. Por exemplo, o mecanismo TJ2P diminuiu a taxa de perda de quadros em $80 \%$ em relação às transmissões pelo BLR e XLinGO. Isso se deve ao fato de que o mecanismo TJ2P escolhe veículos para participar do pelotão que estejam movimentando-se no mesmo sentido, com velocidades semelhantes e uma distância apropriada para disseminar o vídeo, diminuindo as desconexões causadas pela mobilidade dos veículos. O mecanismo TJ2P considera teoria dos jogos para decidir quais veículos devem participar do pelotão e resolve situações de conflitos entre veículos durante a transmissão. Por outro lado, os protocolos XLinGO e BLR não consideram formação de pelotão para disseminação do vídeo e, assim, tais protocolos têm um grande número de desconexões causadas pelo baixo tempo de contato entre os veículos.

Apesar do ganho de SSIM dos vídeos transmitidos pelo mecanismo TJ2P, os veículos que participam do pelotão têm o seu tempo de viagem alterado. Uma vez que os veículos têm de movimentar-se com a mesma velocidade dos demais veículos do pelotão. Sendo assim, os veículos que participaram do pelotão tiveram em média um aumento no tempo de viagem de 11.2 segundos, com variação de 1.6 segundos. Para compensar esse atraso, os veículos receberam uma recompensa por terem participado desse pelotão e encaminhado os pacotes do vídeo. Além disso, tais veículos terão prioridade na transmissão de conteúdo no futuro ou participar de pelotão composto por veículos com melhores reputações e, assim, receber vídeos com qualidade assegurada. Esse tempo pode ser considerado pequeno dado que a colaboração ajudou a transmitir vídeos de situações perigosas em uma estrada e, assim, informar visualmente os motoristas e equipes de resgate sobre o ocorrido. Por outro lado, os veículos não tiveram seu tempo de viagem alterado nas transmissões realizadas pelos protocolos XLinGO e BLR, uma vez que tais protocolos não consideram formação de pelotão ou mudança de velocidade dos veículos.

\footnotetext{
${ }^{3}$ http://www.hbefa.net
} 


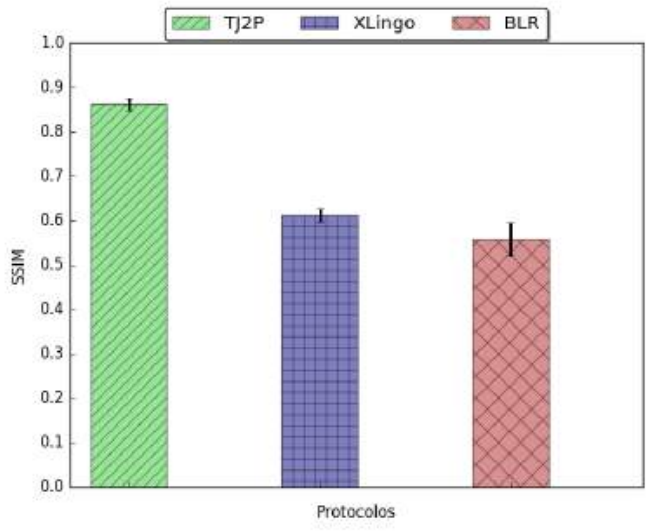

Figura 2. SSIM para os Vídeos Transmitidos

A Figura 3 apresenta a média do VQM para vídeos transmitidos em diferentes distâncias entre os veículos $S N$ e $D N$ pelo TJ2P, BLR e XLinGO. Ao contrário dos resultados de SSIM, quanto menor for o valor do VQM melhor será a qualidade de vídeo. Os resultados de VQM confirmam os benefícios da disseminação de vídeo com suporte a QoE pelo mecanismo TJ2P, o qual considera a formação de pelotão para evitar falhas de comunicação devido à mobilidade dos veículos. Por exemplo, os vídeos transmitidos pelo mecanismo TJ2P tem um VQM 50\% melhor em comparação com as transmissões feitas pelos protocolos BLR e XLinGO. Com base nos resultados SSIM e VQM foi possível confirmar eficiência do mecanismo TJ2P em garantir a transmissão de vídeo em uma VANET com QoE adequado.

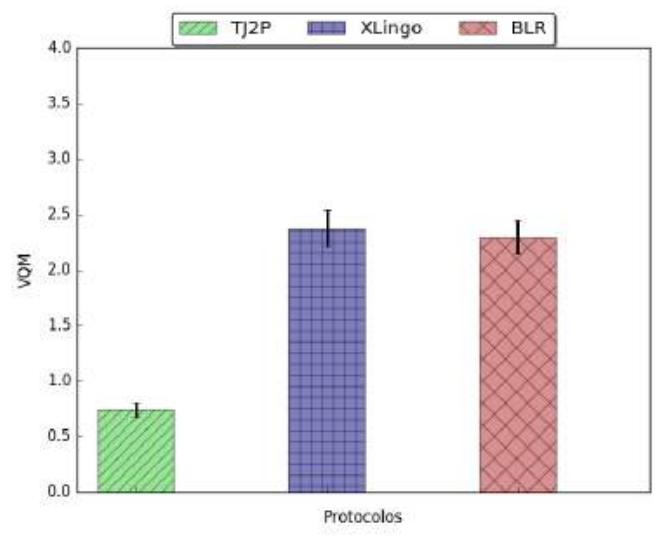

Figura 3. VQM para os Vídeos Transmitidos

Como os veículos do pelotão têm as suas acelerações alteradas, a emissão de $\mathrm{CO}_{2}$ e o consumo de combustível são alterados também. A Figura 4 apresenta o consumo de combustível para os veículos que encaminharam os pacotes de vídeo via TJ2P, BLR e XLinGO. Pode-se observar que os veículos tiveram o mesmo consumo de combustível nos protocolos BLR e XLinGO, pois tais protocolos não consideram alteração na aceleração dos veículos. Sendo assim, esse valor de consumo de combustível serve como referência para analisar o consumo de combustível do mecanismo TJ2P. Ainda é possível notar que o consumo de combustível dos veículos foi reduzido em $32 \%$ ao participar do pelotão, 
considerando o mecanismo TJ2P. Essa redução no consumo de combustível deve-se ao fato de que a velocidade dos veículos foi reduzida para participar do pelotão. Essa redução de velocidade é explicada para reduzir a quantidade de acidentes.

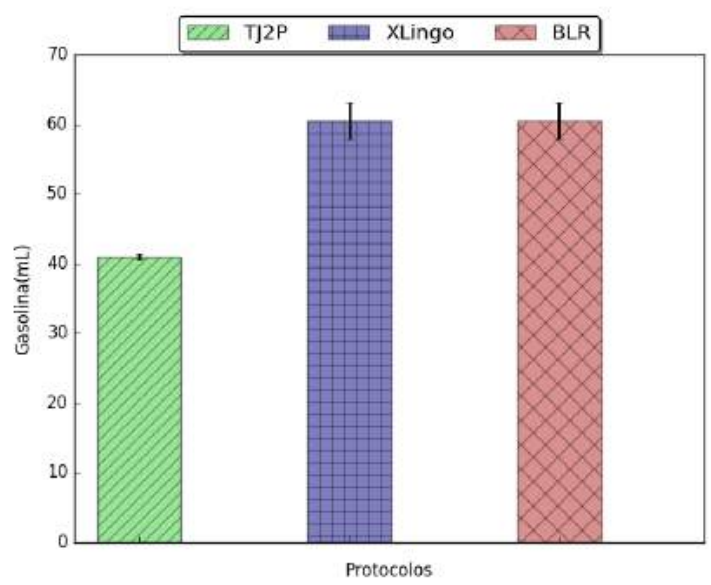

Figura 4. Consumo de Combustível

A Figura 5 apresenta os resultados de emissão de $\mathrm{CO}_{2}$ dos veículos que encaminharam os pacotes de vídeo pelo TJ2P, BLR e XLinGO. Novamente, os protocolos XLinGO e BLR apresentam o mesmo valor de emissão de $\mathrm{CO}_{2}$, pois os veículos não sofrerem alteração na aceleração. Por outro lado, o mecanismo TJ2P diminui a poluição do ar em $31 \%$ comparado ao BLR e XLinGO, devido à redução na velocidade dos veículos.

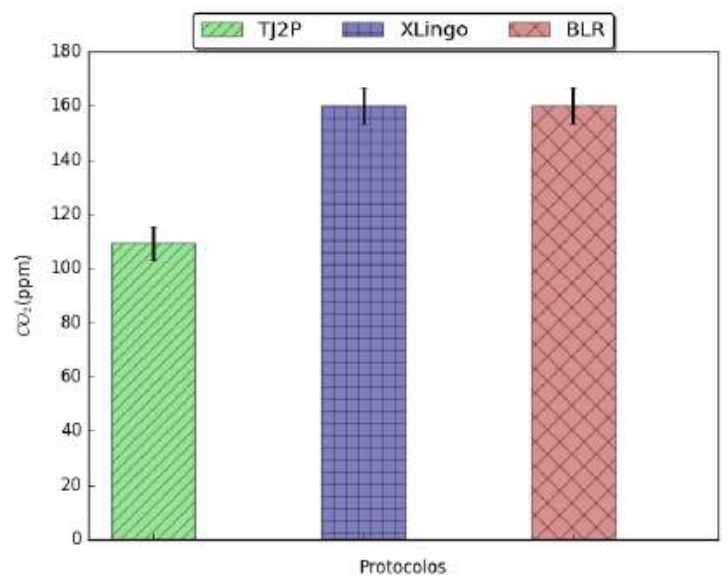

Figura 5. Emissão de $\mathrm{CO}_{2}$

Em relação ao número de mensagens trocadas por cada protocolo, o BLR e XLinGO não incluem mensagens extras para o estabelecimento de rotas por serem protocolos do tipo beaconless, apesar de o XLinGO incluir mensagens para a manutenção de rotas (1 mensagem é enviada a cada 5 pacotes recebido em cada salto [Rosário et al. 2014]). O mecanismo TJ2P considera a troca de pelo menos 3 mensagens a cada salto para a criação do pelotão e não considera troca de mensagem para a manutenção do pelotão. Por outro lado, TJ2P entregou 50\% a mais de vídeos que os pro- 
tocolos XLinGO e BLR, ou seja, o mecanismo TJ2P tem uma maior taxa de sucesso na transmissão de vídeos.

A partir da análise dos resultados, foi possível notar que o mecanismo TJ2P entregou vídeos com QoE 39\% melhores que os vídeos transmitidos pelos protocolos BLR e XLinGO. Apesar dos veículos que participaram do pelotão terem aumentado o seu tempo de viagem em 11 segundos, tais veículos tiveram uma redução na emissão de $\mathrm{CO}_{2}$ e consumo de combustível em $30 \%$, além de terem recebido uma recompensa pela participação da coligação.

\section{Conclusão e Trabalhos Futuros}

Este artigo apresentou o mecanismo TJ2P, o qual provê a formação de pelotão entre veículos de uma VANET utilizando teoria dos jogos para prover disseminação de vídeo com QoE adequado. O mecanismo TJ2P mitiga os problemas relacionados às frequentes desconexões causadas pela mobilidade dos veículos. Para formação do pelotão, foi considerado um jogo não cooperativo e simultâneo entre um conjunto de veículos em movimento no mesmo sentido, com velocidades semelhantes e com distância que possa prover disseminação de vídeo com qualidade. Dessa forma, o mecanismo aumenta a conectividade entre os veículos, provendo transmissão de vídeo com suporte a QoE em uma VANET. Resultados de simulações mostram a eficiência do mecanismo proposto em comparação com protocolos BLR e XLinGO para garantir a transmissão de vídeo com suporte a QoE. Ademais, pode-se constatar que apesar do mecanismo alterar a velocidade dos veículos, o consumo de combustível e emissão de $\mathrm{CO}_{2}$ foram reduzidos. Isso é explicado porque os veículos do pelotão diminuíram a sua velocidade. Como trabalhos futuros, pretende-se aplicar algoritmos de melhor posicionamento dos veículos em conjunto com o mecanismo TJ2P.

\section{Agradecimento}

Este trabalho foi parcialmente financiado com recursos do Conselho Nacional de Desenvolvimento Científico e Tecnológico (CNPq).

\section{Referências}

Amoozadeh, M., Deng, H., Chuah, C.-N., Zhang, H. M., and Ghosal, D. (2015). Platoon Management with Cooperative Adaptive Cruise Control Enabled by VANET. Vehicular Communications, 2(2):110-123.

Braun, T., Heissenbüttel, M., and Roth, T. (2010). Performance of the beacon-less routing protocol in realistic scenarios. Ad Hoc Network, 8(1):96-107.

Chen, T., Wu, L., Wu, F., and Zhong, S. (2011). Stimulating Cooperation in Vehicular Ad Hoc Networks: a Coalitional Game Theoretic Approach. IEEE Transactions on Vehicular Technology, 60(2):566-579.

Cisco (2016). Cisco Visual Networking Index: Forecast and Methodology, 2015-2020. Technical report, Tech Report.

Di Felice, M., Bedogni, L., and Bononi, L. (2013). Group Communication on Highways: An Evaluation Study of Geocast Protocols and Applications. Ad Hoc Networks, 11(3):818-832. 
Ericsson (2013). Ericsson mobility report: On the pulse of the networked society. Technical report, Ericsson, White Paper.

Gerla, M., Wu, C., Pau, G., and Zhu, X. (2014). Content Distribution in VANETs. Vehicular Communications, 1(1):3-12.

Hu, Z., Zheng, Z., Wang, T., Song, L., and Li, X. (2016). Game Theoretic Approaches for Wireless Proactive Caching. IEEE Communications Magazine, 54(8):37-43.

Jia, D., Lu, K., and Wang, J. (2014). On the Network Connectivity of Platoon-based Vehicular Cyber-physical Systems. Transportation Research Part C: Emerging Technologies, 40:215-230.

Lee, E., Lee, E.-K., Gerla, M., and Oh, S. Y. (2014). Vehicular Cloud Networking: Architecture and Design Principles. IEEE Communications Magazine, 52(2):148-155.

Library, V. (2017). YUV Video Sequence Library. http://trace.eas.asu.edu/yuv/ Mar. 2017.

Liotou, E., Tsolkas, D., Passas, N., and Merakos, L. (2015). Quality of Experience Management in Mobile Cellular Networks: Key Issues and Design Challenges. IEEE Communications Magazine, 53(7):145-153.

Liu, J., Wan, J., Wang, Q., Deng, P., Zhou, K., and Qiao, Y. (2016). A Survey on Position-based Routing for Vehicular Ad Hoc Networks. Telecommunication Systems, 62(1):15-30.

Quadros, C., Santos, A., Gerla, M., and Cerqueira, E. (2015). A QoE-Aware Mechanism to Improve the Dissemination of Live Videos over VANETs. In Proceedings of the XXXIII Brazilian Symposium on Computer Networks and Distributed Systems (SBRC 2015), pages 31-40. IEEE.

Rosário, D., Zhao, Z., Santos, A., Braun, T., and Cerqueira, E. (2014). A beaconless Opportunistic Routing based on a Cross-layer Approach for Efficient Video Dissemination in Mobile Multimedia IoT Applications. Computer communications, 45(1):2131 .

Torres, A., Calafate, C. T., Cano, J.-C., Manzoni, P., and Ji, Y. (2015). Evaluation of Flooding Schemes for Real-time Video Transmission in VANETs. Ad Hoc Networks, 24:3-20.

Uppoor, S. and Fiore, M. (2012). Insights on Metropolitan-scale Vehicular Mobility from a Networking Perspective. In Proceedings of the 4th ACM international workshop on Hot topics in planet-scale measurement, pages 39-44. ACM.

Wang, Y. and Lin, X. (2015). User-provided Networking for QoE Provisioning in Mobile Networks. IEEE Wireless Communications, 22(4):26-33.

Zhang, Y. and Cao, G. (2011). V-pada: Vehicle-platoon-aware data access in vanets. IEEE Transactions on Vehicular Technology, 60(5):2326-2339. 